Original Article

\title{
Effect of the frequency of therapy on the performance of activities of daily living in children with cerebral palsy
}

\author{
Eun-Young PARK, PT, PhD ${ }^{1)}$, Eun-Joo KIM, OT, $\mathrm{PhD}^{2)^{*}}$ \\ 1) Department of Secondary Special Education, College of Education, Jeonju University, \\ Republic of Korea \\ 2) Department of Occupational Therapy, College of Medical Sciences, Jeonju University: \\ 1200 3-ga, Hyoja-dong, Wansan-gu, Jeonju 560-759, Republic of Korea
}

\begin{abstract}
Purpose] This study investigated the frequency effect of physical and occupational therapy on activities of daily living performance in children with cerebral palsy. [Subjects and Methods] A total of 162 children with cerebral palsy who attended a convalescent or rehabilitation center for disabled individuals or a special school for physical disabilities in South Korea participated in this study. The Pediatric Evaluation Disability Inventory was used to collect data on activities of daily living performance according to physical therapy frequency based on neurodevelopmental therapy for 1 year. [Results] The relationships between physical therapy frequency and activities of daily living performance (mobility, social function, and total functional skill) and between occupational therapy frequency and activities of daily living performance (social function and total functional skill) were significant. There was no significant difference in activities of daily living performance according to physical therapy frequency. The difference in the activities of daily living performance according to occupational therapy frequency was significant for social function. [Conclusion] Intensive occupational therapy was more effective in improving activities of daily living performance in children with cerebral palsy. In particular, their social function further improved with intensive physical therapy.

Key words: Children with cerebral palsy, Frequency of therapy, Activities of daily living
\end{abstract}

(This article was submitted Nov. 20, 2017, and was accepted Feb. 27, 2018)

\section{INTRODUCTION}

The intensity effect of therapies has been examined among patients with stroke, back pain, and traumatic brain injuries (TBIs). It was verified that the intensity effect of therapies was mainly observed in patients with stroke. A higher therapy intensity was associated with a shorter length of stay and improvement in functional independence in such patients ${ }^{1)}$; further, higher physical therapy (PT) and occupational therapy (OT) intensities increased the possibility of improving mobility and functional independence in activities of daily living (ADL). A synthesis research including nine controlled studies reported that there was a small but a statistically significant intensity effect relationship of PT and OT with improvement in ADL performance and functional outcome parameters in patients with stroke ${ }^{2)}$. The study also examined the relationship between therapy intensity and rehabilitation outcomes after TBIs and showed that therapy intensity was predictive of the motor function at discharge ${ }^{3)}$.

Despite the importance of appropriate treatment frequency and duration of therapy services in determining government financial and insurance benefits and improving the functional abilities during pediatric PT, evidence for determining the optimal program is lacking ${ }^{4,5)}$. Recently, the guidelines regarding the frequency and duration of therapy for pediatric patients

*Corresponding author. Eun-Joo Kim (E-mail: kimot@jj.ac.kr)

(C2018 The Society of Physical Therapy Science. Published by IPEC Inc.

(c) $(-)$ This is an open-access article distributed under the terms of the Creative Commons Attribution Non-Commercial No Derivatives

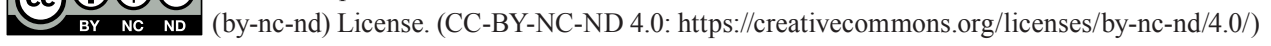


have been reported. The treatment frequency guidelines (TFGs) developed by Bailes et al. ${ }^{6}$ ) could be used in deciding the treatment frequency in pediatric settings. The TFGs provide a good starting point for decision making regarding treatment frequency for pediatric patients ${ }^{7}$ ). The TFGs were also used across inpatient and outpatient settings, and four modes were developed as follows. First, the intensive mode involves a frequency of 3 to 11 times a week; the weekly or bimonthly mode, 1 to 2 times a week or every other week; the periodic mode, once per month or less often but at regularly scheduled intervals; and the consultative mode, episodic or as needed.

Children with chronic disabilities, such as cerebral palsy (CP), receive therapy for as long as the family requests services ${ }^{6}$. Although the frequency of therapy might change owing to family complaints, government financial and insurance benefits are also important factors in the decision making of therapists regarding therapy frequency. For that reason, evidence of therapy effectiveness according to varying frequency modes is needed. Moreover, the differences according to different functional outcomes should be verified using evidence in order to decide on the therapy frequency. Among functional outcomes, a relationship between gross motor function and intensity of therapy has been reported ${ }^{8)}$. Intensive PT was more effective in improving the gross motor function of children with $\mathrm{CP}$, especially crawling and kneeling abilities; further, the standing ability further improved with intensive PT. However, the relationship between ADL performance and therapy frequency has not been examined yet. Thus, the purpose of this study was to examine the relationship between ADL performance and therapy frequency.

\section{SUBJECTS AND METHODS}

The study sample comprised 162 children with CP (mean age: 8 years and 1 month; SD: 3 years and 5 months) who attended a convalescent or rehabilitation center for disabled individuals or a special school for physical disabilities in South Korea. A total of 91 boys (56.2\%) and 71 girls (43.8\%) were recruited in this study. The age range was 3 to 15 years. The parents of all children agreed to participate in this study. This study was approved by the Ethics Committee of Jeonju University (Jeonju University IRB-1). Written informed consent to participate in the study was obtained from the parents of all children. The types of CP in the children were spastic (79.6\%), dyskinetic/athetotic $(9.9 \%)$, ataxic $(4.3 \%)$, and hypotonic $(6.2 \%)$. The participants were classified using the Gross Motor Function Classification System; 23 (14.2\%) were classified into Level I, 30 (18.5\%) into Level II, 23 (14.2\%) into Level III, 21 (13.0\%) into Level IV, and 65 (40.1\%) into Level V.

To evaluate ADL performance, the functional skill domain in the Pediatric Evaluation of Disability Inventory (PEDI), which was developed in the USA by Haley et al. was used. The functional skill domain consists of three categories of selfcare, mobility, and social function and 197 specific questions. Each child was scored either with 1 point for being capable of performing the assigned task or 0 point for incomplete performance and being unable to perform the task. The one-sample Kolmogorov-Smirnov (K-S) test was employed to test for the normal distribution of data. Because the results of the K-S test were statistically significant, a non-parametric test was performed to verify the effect of the frequency of PT on ADL performance. The Spearman correlation coefficient and Kruskal-Wallis test were also used. The Tukey test using ranks was employed as a post hoc test.

\section{RESULTS}

Table 1 shows the correlation coefficients between the frequency of therapy and ADL performance measured by the PEDI functional skill, including self-care, mobility, and social function. There were significant correlations between the frequency of PT and mobility, social function, and total PEDI functional skill. Similarly, the correlation of the frequency of OT with social function and total PEDI functional skill was significant.

The results of the Kruskal-Wallis test are presented in Table 2. The differences in the ADL performance according to the frequency of PT were not significant in all domains and total PEDI functional skill; that according to the frequency of OT was significant in the total PEDI functional skill. The intensive mode of OT showed a significant effect of improving the PEDI functional skill compared with the bimonthly mode.

\section{DISCUSSION}

ADL performance is one of the major goals of PT and OT in rehabilitation settings ${ }^{9)}$. The evidence for a relationship between the frequency of PT and OT and ADL performance should be clarified to determine the proper frequency of therapy for the improvement of ADL performance in children with CP. In this study, the relationship between the frequency of therapy and ADL performance, measured using the PEDI functional skill scale, was investigated during a 1 year follow-up.

Mobility, social function, and total PEDI functional skill showed significant correlations with the frequency of PT. However, the correlation between mobility and the frequency of OT was not significant. In patients with stroke, a significant relationship between mobility and therapy intensity (including PT and OT) was reported ${ }^{1}$. A previous meta-analysis demonstrated a statistically significant summary effect size for ADL performance $(0.28 \pm 0.12)$ in patients with stroke ${ }^{2}$. In patients with TBIs, the therapy intensity was predictive of motor functioning at discharge ${ }^{3)}$. In children with CP, an intensive PT might be effective in improving gross motor function, and the intensity effect was greater in crawling and kneeling and standing 
Table 1. The correlation between frequency of therapy and change of activities of daily living

\begin{tabular}{lcccc}
\hline \multicolumn{1}{c}{ Category } & Self-care & Mobility & Social function & PEDI $^{\text {a }}$ Functional skill total \\
\hline Frequency of physical therapy & 0.149 & $0.163^{*}$ & $0.228^{* *}$ & $0.196^{*}$ \\
Frequency of occupational therapy & 0.149 & 0.141 & $0.219^{* *}$ & $0.226^{* *}$ \\
\hline
\end{tabular}

$* \mathrm{p}<0.05, * * \mathrm{p}<0.01$.

aPEDI: Pediatric Evaluation of Disability Inventory.

Table 2. The differences of the change of activities of daily living according to frequency of therapy

\begin{tabular}{|c|c|c|c|c|c|}
\hline \multicolumn{2}{|c|}{ Category } & \multicolumn{2}{|c|}{ Physical therapy } & \multicolumn{2}{|c|}{ Occupational therapy } \\
\hline PEDI $^{a}$ Functional skill domain & Frequency of therapy & Mean & SD & Mean & SD \\
\hline \multirow[t]{3}{*}{ Self-care } & Consultative & 0.80 & 3.51 & 0.62 & 10.67 \\
\hline & Bimonthly & -0.54 & 13.83 & 0.14 & 13.87 \\
\hline & Intensive & 3.36 & 14.18 & 4.70 & 15.28 \\
\hline \multirow[t]{3}{*}{ Mobility } & Consultative & 0.64 & 7.13 & 3.66 & 15.57 \\
\hline & Bimonthly & 1.75 & 13.42 & 1.86 & 13.86 \\
\hline & Intensive & 4.61 & 15.35 & 5.33 & 13.77 \\
\hline \multirow[t]{3}{*}{ Social function } & Consultative & 0.96 & 7.62 & -1.84 & 17.02 \\
\hline & Bimonthly & -0.52 & 12.72 & -1.18 & 14.87 \\
\hline & Intensive & 1.32 & 18.88 & 5.93 & 15.57 \\
\hline \multirow[t]{3}{*}{ PEDI $^{\mathrm{a}}$ Functional skill total } & Consultative & 0.82 & 4.68 & $0.82^{\mathrm{ab}}$ & 12.99 \\
\hline & Bimonthly & 0.40 & 11.54 & $0.42^{\mathrm{a}}$ & 11.80 \\
\hline & Intensive & 3.11 & 14.03 & $5.42^{\mathrm{b}}$ & 13.60 \\
\hline
\end{tabular}

aPEDI: Pediatric Evaluation of Disability Inventory.

The different superscript letter means the significant difference $(\mathrm{p}<0.05)$.

abilities ${ }^{8)}$. However, some studies reported that the therapy intensity did not have a significant effect on the functional status of patients with TBIs. A multiple regression analysis was performed to verify the relationships between functional status at discharge and intensity of therapies received during inpatient medical rehabilitation. The results showed that the intensities of OT, PT, and speech therapy were not significant predictors of outcomes for either group, controlling linearly for admission function, psychology intensity, length of stay, onset to admission interval, age, and interrupted stays ${ }^{10)}$. More recent studies on patients with TBIs supported the non-significant effect of therapy intensity ${ }^{11)}$. The controversial results regarding therapy intensity effect indicate that several factors could affect the outcomes besides therapy intensity. Therefore, assertive conclusions could not be made by a single situation, and several factors, such as diagnosis, type of therapy, and severity of symptoms, should be considered in the decision making for therapy intensity.

Similar to the correlation results, only the intensive mode of OT showed a significant effect of improving the PEDI functional skill level compared to that in the bimonthly mode. The intensive mode includes a wide range of therapy frequencies, that is, from 3 to 11 times a week. To accumulate evidence on decision making for the appropriate therapy frequency, the type of mode was developed more specifically.

Although this study suggests that robust PT and OT for ADL performance improvement in children with CP is effective, on the basis of 2 years of data collection, there is still insufficient clinical evidence for the relationship between treatment intensity and efficacy. Future research studies are needed to investigate the relationship between various treatment intensities and outcomes in children with CP to provide meaningful information for decision making in clinical settings.

This study examined the effect of PT and OT according to frequency during a 1 year follow-up of ADL performance in children with CP. The therapy frequency showed a significant correlation with ADL performance; particularly, intensive OT was more effective in improving ADL performance.

\section{Conflict of interest}

None.

\section{REFERENCES}

1) Jette DU, Warren RL, Wirtalla C: The relation between therapy intensity and outcomes of rehabilitation in skilled nursing facilities. Arch Phys Med Rehabil, 2005, 86: 373-379. [Medline] [CrossRef] 
2) Kwakkel G, Wagenaar RC, Koelman TW, et al.: Effects of intensity of rehabilitation after stroke. A research synthesis. Stroke, 1997, 28: 1550-1556. [Medline] [CrossRef]

3) Cifu DX, Kreutzer JS, Kolakowsky-Hayner SA, et al.: The relationship between therapy intensity and rehabilitative outcomes after traumatic brain injury: a multicenter analysis. Arch Phys Med Rehabil, 2003, 84: 1441-1448. [Medline] [CrossRef]

4) Smith BA, Fields CJ, Fernandez N: Physical therapists make accurate and appropriate discharge recommendations for patients who are acutely ill. Phys Ther, 2010, 90: 693-703. [Medline] [CrossRef]

5) Palisano RJ, Murr S: Intensity of therapy services: what are the considerations? Phys Occup Ther Pediatr, 2009, 29: 107-112. [Medline] [CrossRef]

6) Bailes AF, Reder R, Burch C: Development of guidelines for determining frequency of therapy services in a pediatric medical setting. Pediatr Phys Ther, 2008, 20: 194-198. [Medline] [CrossRef]

7) Hanson H, Harrington AT, Nixon-Cave K: Implementing treatment frequency and duration guidelines in a hospital-based pediatric outpatient setting: administrative case report. Phys Ther, 2015, 95: 678-684. [Medline] [CrossRef]

8) Tsorlakis N, Evaggelinou C, Grouios G, et al.: Effect of intensive neurodevelopmental treatment in gross motor function of children with cerebral palsy. Dev Med Child Neurol, 2004, 46: 740-745. [Medline] [CrossRef]

9) Kim WH, Park EY: Causal relation between spasticity, strength, gross motor function, and functional outcome in children with cerebral palsy: a path analysis. Dev Med Child Neurol, 2011, 53: 68-73. [Medline] [CrossRef]

10) Heinemann AW, Hamilton B, Linacre JM, et al.: Functional status and therapeutic intensity during inpatient rehabilitation. Am J Phys Med Rehabil, 1995, 74 : 315-326. [Medline] [CrossRef]

11) Hart T, Whyte J, Poulsen I, et al.: How do intensity and duration of rehabilitation services affect outcomes from severe traumatic brain injury? A natural experiment comparing health care delivery systems in 2 developed nations. Arch Phys Med Rehabil, 2016, 97: 2045-2053. [Medline] [CrossRef] 